\title{
Study on Energy Dissipation Law in the Process of Coal and Gas Outburst
}

\author{
Shugang $\mathrm{Li}^{1, \mathrm{a}}$, Ning Wang ${ }^{1, \mathrm{~b}^{*}}$ and Tianjun Zhang ${ }^{2, \mathrm{c}}$ \\ ${ }^{1}$ College of Energy Science and Engineering, Xi'an University of Science \& Technology, Xi'an, \\ Shaanxi, 710054, China \\ ${ }^{2}$ School of Science, Xi'an University of Science \& Technology, Xi'an, Shaanxi, 710054, China \\ alisg@.xust.edu.cn, bwangningxust@163.com, ctianjun_zhang@126.com
}

Keywords: Coal and gas outburst; Energy; Dissipation law; Transfer process.

\begin{abstract}
Research from the angle of energy is one of the important way to explore the cause of coal and gas outburst for grasping the law of coal and gas outburst. (Objective): In order to study energy dissipation law in the process of coal and gas outburst. (Methods): Build the relationship between strain and stress of coal and rock according to the physical equations of elastic mechanics, obtain the deformation potential energy of the coal and rock without considering the change of gas pressure and temperature, establish the practical dynamics equation of gas in coal seams. (Results): The research results show that gas rich coal and rock stores a great deal of deformation energy, adsorption energy of gas adsorbing on coal, gas expansion energy and gas fluid potential energy, etc. (Conclusion): These energy can make coal broken in coal and rock damage fracture process and provide continuous driving energy for coal and gas outburst.
\end{abstract}

\section{Introduction}

Coal and gas outburst is a dynamic process in which coal exposed surface suddenly and continuously flying to roadway space in a relatively short time[1-3], continuous coal and gas outburst needs a lot of energy to provide power, so the research from the angle of energy is one of the important way to explore the cause of coal and gas outburst for grasping the law of coal and gas outburst[4-6]. In order to study the total energy dissipation law in the process of coal and gas outburst, analyze the energy change process of one micro coal unit in different stages[7, 8].

In a stable state, energy saving in coal and rock mass includes deformation potential energy influenced by crustal stress, adsorption energy of gas adsorbing on coal and dissociative gas flow energy stored in coal and the pores[9-11]. To study the energy dissipation law in coal and gas outburst is actually to explore the consumption and transfer process of the three kinds of energy.

\section{Deformation Potential Energy of Coal and Rock}

According to triaxial stress test, coal and rock can be seen as elastomer before damage. In the stress stage of coal and gas outburst process, that is, in the original stress state of coal and rock, the stress of the micro unit can be treated as in equilibrium state. In the stress concentration stage of coal and gas outburst, micro coal units produce stress concentration in a certain direction, resulting in changing stress of micro coal units, so focus direction stress decreases and tangential stress of the other two directions increase. The change of stress effects little on the small pore volume of the original coal body, because of adsorption gas existing in the small pores, stress changing also influences little to gas pressure within the pores, the increase of the stress just makes deformation potential energy increase of coal skeleton. Therefore, it is reasonable to consider no change of gas pressure while considering deformation potential energy of coal and rock. Crustal stress makes deformation potential energy of coal and rock increase in the particles stress concentration, If the micro units of coal are seen as a closed thermodynamic system, and ignore the temperature change of the gas pressure in the process of stress concentration, the work done by coal strata is equal to the increasing of elastic energy 
of coal micro units. In balance state, both the kinetic energy and the mechanical energy of the coal and rock have not changed, so the decrease of the potential energy is completely transformed into deformation potential energy storing in the internal of coal and rock. To sum up, deformation potential energy of coal and rock without considering the change of the gas pressure and temperature can be expressed as Eq. 1.

$$
E_{\varepsilon}=\frac{1}{2} \iiint\left(\sigma_{x} \varepsilon_{x}+\sigma_{y} \varepsilon_{y}+\sigma_{z} \varepsilon_{z}+\gamma_{x y} \tau_{x y}+\gamma_{x y} \tau_{x y}+\gamma_{y z} \tau_{y z}+\gamma_{z x} \tau_{z x}\right) \mathrm{d} x \mathrm{~d} y \mathrm{~d} z
$$

In Eq. $1, \mu$ stands for poisson's ratio of coal, $\sigma_{x} 、 \sigma_{y} 、 \sigma_{z}$ mean the stress of coal vertical coordinates $x, y, z$ directions, $N \cdot m^{-2}, \varepsilon_{x} 、 \varepsilon_{y} 、 \varepsilon_{z}$ mean the strain of coal vertical coordinates $x, y, z$ directions. Obtaining the relationship of strain and stress according to the physical equations of elastic mechanics shown as Eq. 2.

$$
\left.\begin{array}{cc}
\sigma_{x}=\frac{1}{E}\left[\sigma_{x}-\mu\left(\sigma_{y}+\sigma_{z}\right)\right] & ; \gamma_{x y}=\frac{1}{G} \tau_{x y} \\
\sigma_{y}=\frac{1}{E}\left[\sigma_{y}-\mu\left(\sigma_{x}+\sigma_{z}\right)\right] & ; \gamma_{y z}=\frac{1}{G} \tau_{y z} \\
\sigma_{z}=\frac{1}{E}\left[\sigma_{z}-\mu\left(\sigma_{y}+\sigma_{z}\right)\right] & ; \gamma_{z x}=\frac{1}{G} \tau_{z x}
\end{array}\right\}
$$

In Eq. 2, $\tau_{x y} 、 \tau_{z x} 、 \tau_{y z}$ stand for coal shear stresses, $N \cdot m^{-2}, \gamma_{x y} 、 \gamma_{z x} 、 \gamma_{y z}$ are shear strains of coal. Assume that gas absorbed to coal is applied to coal and rock in uniform, the volume deformation energy of coal element effected by the nominal stress $\sigma_{0}\left(N \cdot m^{-2}\right)$ and adsorption pressure $P_{0}\left(N \cdot m^{-2}\right)$ combining Eq. 1 and Eq. 2 is defined as Eq. 3.

$$
\mathrm{E}_{\varepsilon}=\frac{1-2 \mu}{6 E} \iiint_{V}\left(\sigma_{0}+P_{0}\right)^{2} \mathrm{~d} x \mathrm{~d} y \mathrm{~d} z
$$

In the coal burst phase, micro units of coal are forward to the exposed surface as the stress peak moves, tangential stress and radial stress effected on the micro units of coal are smaller and smaller, so deformation potential energy of coal micro units also gradually reduces. Decreasing of the deformation potential energy means that deformation potential energy the micro unit of coal is consumed, consumed deformation potential energy is converted into two forms: one is coal breakage under the effect of shear stress, and deformation potential energy is mainly transformed into coal and gas kinetic energy, the other one is the heat generated from the friction of coal particles on the crack surface, and partial deformation potential energy will be transformed into heat energy of coal micro units. Fig. 1 shows the energy distribution in the tunnel when the damage volumes of original coal and rock are respectively1.0 $\mathrm{V}$ and $1.5 \mathrm{~V}$.

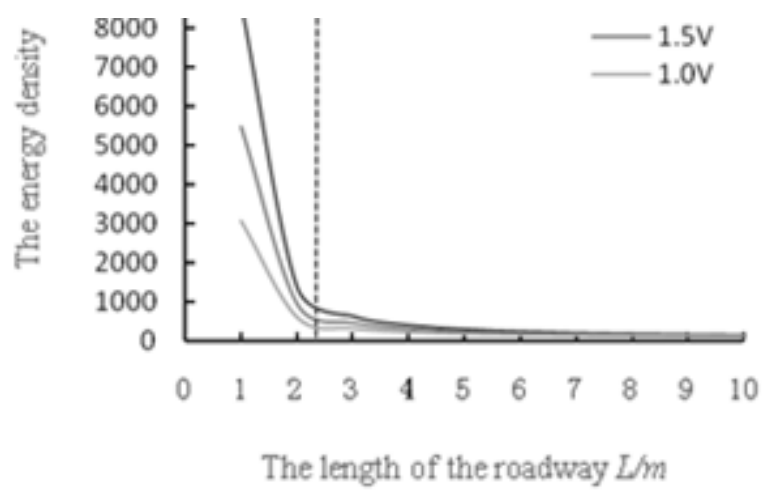

Figure 1. Energy density changing with length of roadway in the roadway 


\section{Adsorption Energy of Gas Adsorbing on Coal}

The coal mine gas mainly comes from coal seams and coal strata, a large number of gas is produced in the process of plants forming into coal, and gas is associated product in the process of coalification. Coal and gas outburst is a very complicated process, in the many factors causing coal and gas outburst, large amounts of gas desorption is an important factor.

Gas adsorption occurs on coal and rock surface and holes, molecules density of gas fluid on the adsorption surface is higher than gas density of free fluid in the roadway. When the adsorption of gas molecules and coal molecules reaches to geometric equilibrium, the calculation formula of adsorption ability can be expressed as Eq. 4.

$$
E_{\text {ads }}=E_{\mathrm{M}}+E_{\mathrm{O}}-E_{\mathrm{O} / \mathrm{M}}
$$

In Eq. $4, E_{a d s}$ is adsorption energy when gas molecular adsorption on coal surface reaches equilibrium state, $E_{M}$ is the energy of coal surface molecular fragment before gas adsorption, $E_{\mathrm{O}}$ is the energy of gas molecular before adsorption, $E_{\mathrm{O} / \mathrm{M}}$ is the total energy of the adsorption system after gas molecular absorbed to coal surface molecular fragment.

\section{Potential Energy of Gas Flow within the Coal and Rock}

Adsorption state gas in coal and rock mainly exists in micro holes and small holes, free state gas mainly exists in the coal and rock mesopores, large pores and fracture. These free state gas is approximately regarded as an ideal gas in the practical engineering, and there are big differences between adsorption gas and free gas in micro holes and small holes, which have on characteristics of an ideal gas. Gas desorption in micro holes and small holes and its transmission way to mesopores, large pores and fracture are very complex, free gas under the effect of pressure gradient can be seen as linear seepage flow to study its delivery process, and then establish the practical dynamic equations of the gas in coal seam.

Combined with the Langmuir equation of gas adsorption on coal, the differential form of gas flow potential energy in coal and rock per unit volume can be expressed as Eq. 5.

$$
\mathrm{d} E_{f l o}=\mathrm{M} v \mathrm{~d} v=\frac{\rho_{2} a b}{\rho_{1}(1+b p)^{2}} v \mathrm{~d} v
$$

In Eq. 5, $v$ is gas flow velocity, $\mathrm{m} / \mathrm{s}, \mathrm{p}$ is free gas pressure, $\mathrm{Pa}, \mathrm{M}$ is gas molar mass, $\mathrm{g} / \mathrm{mol}$, $\rho_{2}$ is the density of coal and rock, $\mathrm{kg} / \mathrm{m}^{3}, \rho_{1}$ is the gas density with pressure for $p$ and temperature for $T, \mathrm{~kg} / \mathrm{m}^{3}$.

Because of gas compressibility, generally the gas flow in coal seam is approximate to linear seepage, that is, gas flow is directly proportional to the gradient of gas pressure $p$ in coal seam, and in accordance with Darcy's law. Stress and temperature field influences the gas movement, penetration ability of pore-fracture media depends on the number of pores and fractures on flow direction, width, connectivity and differential pressure at the ends of the pores and fractures. The permeability of porosity and fracture is derived from the laminar flow equation of flow through the slit. Thus, the equation of gas motion influenced by stress field and temperature field is expressed as Eq. 6 .

$$
v=-\frac{(1+T)^{\mathrm{m}}}{\eta} \mathrm{e}^{\mathrm{-a} \sigma} \mathbf{K} \cdot \nabla \tilde{P}
$$

In Eq. 6, $\eta$ means gas dynamic viscosity, $\mathrm{kg} /(\mathrm{m} \cdot \mathrm{s}), K$ is the seepage coefficient of coal seam (a third-order tensor), $m$ and $a$ are parameters constants, $T$ is free gas temperature, $K, \nabla$ is the Hamiltonian operator, $\nabla=\mathrm{i}^{*} \mathrm{~d} / \mathrm{dx}+\mathrm{j} * \mathrm{~d} / \mathrm{dy}+\mathrm{k}^{*} \mathrm{~d} / \mathrm{dz}, \mathbf{K} \cdot \nabla P^{\prime}$ stands for gas pressure gradient in the flow direction of coal and rock.

Combining Eq. 5 with Eq. 6, gas flow potential energy in coal and rock is shown as Eq. 7.

$$
E_{f l o}=\int \frac{\rho_{2} a b}{\rho_{1}(1+b p)^{2}} v \mathrm{~d} v=\frac{1}{2} \int \frac{\rho_{2} a b}{\rho_{1}(1+b p)^{2}}\left(-\frac{(1+T)^{\mathrm{m}}}{\eta} \mathrm{e}^{\mathrm{e} \sigma}\right)^{2} \mathrm{~d}|\mathbf{K} \cdot \nabla \tilde{P}|^{2}
$$

In practical engineering, as gas discharge is slow, at the same time free phase and adsorption state gas exist heat exchange with coal body, the thermal change process of gas in coal seams can be similar 
as isothermal process. When gas has no emission in the original coal seams, adsorption state gas and free phase gas are in the thermodynamic equilibrium state, and their macro velocities are zeros, with the same pressure, temperature and chemical potential, so the mass and energy of the system of coal and rock can be thought of on diffusion. According to Eq. 7, the gas flow potential energy is zero in coal and rock, that is, $\widetilde{E}_{f l o}=0$.

Due to the adsorption potential energy of adsorption phase gas is greater than that of free state gas, physical border conditions of gas emission in coal seam changes, the original stress state and thermodynamic equilibrium state in coal seams are destroyed. When the gas pressure in coal seam decreases gradually, free gas flow in mesopores, large pores and fracture under the effect of pressure gradient, temperature decreases with the decreasing pressure.

\section{Conclusions}

With coal micro unit as the research object, analyze the energy change process of one micro coal unit in different stages to study the total energy dissipation law in the process of coal and gas outburst.

Deformation potential energy of coal and rock is converted into two forms: one is coal breakage under the effect of shear stress, and deformation potential energy is mainly transformed into coal and gas kinetic energy, the other one is the heat generated from the friction of coal particles on the crack surface, and partial deformation potential energy will be transformed into heat energy of coal micro units.

Adsorption potential energy of adsorption phase gas is greater than that of free state gas, physical border conditions of gas emission in coal seam changes, the original stress state and thermodynamic equilibrium state in coal seams are destroyed. When the gas pressure in coal seam decreases gradually, free gas flow in mesopores, large pores and fracture under the effect of pressure gradient, temperature decreases with the decreasing pressure.

Gas rich coal and rock stores a great deal of deformation energy, adsorption energy of gas adsorbing on coal, gas expansion energy and gas fluid potential energy, etc. These energy can make coal broken in coal and rock damage fracture process and provide continuous driving energy for coal and gas outburst.

\section{Acknowledgements}

This work was carried out in the context of the Project NO. 51327007, 51174157, 51374168 supported by the National Natural Science Foundation of China (NSFC). The financial support is greatly acknowledged.

\section{References}

[1] XIONG Yangtao, HUANG Gun, LUO Jiayuan, et al. Theoretical Anslysis and Experimental Study of Energy Dissipation of Coal and Gas Outburst[J]. Chinese Journal of Rock Mechanics and Engineering, 34(2): 3694-3702. (2015)

[2] WANG Gang, WU Mengmeng, WANG Haiyang, et al. Sensitivity Analysis of Factors Affecting Coal and Gas Outburst Based on A Energy Equilibrium Model[J]. Chinese Journal of Rock Mechanics and Engineering, 34(2): 238-248. (2015)

[3] KANG Xiangtao, HUANG Gun, SONG Zhenlong, et al. Research on Characteristics of Energy Dissipation and Seepage of Coal Containing Gas under Triaxial Compression[J]. Rock and Soil Mechanics and Engineering, 36(3): 762-768. (2015)

[4] JIANG Chenglin, YU Qixiang. Rules of Energy Dissipation in Coal and Gas Outburst[J]. Journal of China Coal Society, 21(2): 173-178. (1996) 
[5] WANG Wen, LI Huamin, GU Helong, et al. Feature Analysis of Energy Dissipation of Water-saturated Coal Samples under Coupled Static-dynamic Loads[J]. Chinese Journal of Rock Mechanics and Engineering, 34(2): 3965-3971. (2015)

[6] TU Qing-yi, CHENG Yuanping, WANG Liang, et al. Study on Dynamic Process of Coal and Gas Outburst[J]. Coal Science and Technology, 43(6): 71-75. (2015)

[7] CAI Cheng-gong, WANG You-an. Qualitative and Quantitative Analysis of General Regularity of Coal and Gas Outburst[J]. China Safety Science Journal, 14(6):109-112. (2004)

[8] CHEN Yun, JIN Yan, CHEN Mian. A Rock Brittleness Evaluation Method Based on Energy Dissipation[J]. Chinese Journal of Theoretical and Applied Mechanics, 47(6): 984-993. (2015)

[9] SONG Zhenlong, HAN Peibo, LI Wenpu, et al. Impact of Energy Dissipation of Coal Samples with Rockburst Tendency from Gas in Its Failure Process[J]. Journal of China Coal Society, 40(4): 843-849. (2015)

[10]ZHANG Hao, GAO Jianning, CUI Yongjie. Thermodynamic Analysis of Gas Expansion Energy Calculation Method[J]. Safety in Coal Mines, 46(3): 145-148. (2015)

[11]LI Shugang. Movement of the Surrounding Rock and Gas Delivery in Fully-mechanized Top Coal Caving[M]. Xuzhou: China University of Mining and Technology Press. (2000) 